\title{
Upregulation of PREX2 promotes the proliferation and migration of hepatocellular carcinoma cells via PTEN-AKT signaling
}

\author{
SHUJIE HE ${ }^{1}$, JUAN LIN ${ }^{2}$, SHAOPING YU ${ }^{3}$ and SHIJIE SUN ${ }^{3}$ \\ ${ }^{1}$ Department of Hepatobiliary Surgery, Yantai Mountain Hospital; ${ }^{2}$ Department of Hepatobiliary Surgery, \\ Government Hospital of Yantai City, Yantai, Shandong 264000; ${ }^{3}$ Department of Hepatobiliary Surgery, \\ Yuhuangding Hospital, Yantai, Shandong 264001, P.R. China
}

Received February 20, 2015; Accepted December 15, 2015

DOI: $10.3892 / \mathrm{ol} .2016 .4164$

\begin{abstract}
Phosphatidylinositol-3,4,5-trisphosphate Rac exchanger 2 (PREX2), a regulator of the small guanosine triphosphatase Rac, demonstrates an inhibitory effect on the activity of phosphatase and tensin homolog (PTEN). Previously, PREX2 was implicated in the regulation of cell invasion in hepatocellular carcinoma (HCC). However, the exact role of PREX2 in the regulation of HCC cell proliferation and migration, as well as the underlying mechanisms, remains unclear. In the present study, reverse transcription-quantitative polymerase chain reaction revealed that PREX2 was upregulated in HCC tissue compared with matched adjacent non-tumorous tissue. In addition, the present study demonstrated that the messenger RNA and protein levels of PREX2 increased in human HCC HepG2, LH86, LMH and PLHC-1 cell lines compared with normal human liver THLE-3 cells. Overexpression of PREX2 significantly enhanced the proliferation and migration of HCC cells, and knockdown of PREX2 expression significantly suppressed the proliferation and migration of HCC cells. Additional investigation revealed that overexpression of PREX2 suppressed the activity of PTEN, leading to an enhancement in the activity of protein kinase B (AKT). By contrast, knockdown of PREX2 expression upregulated the activity of PTEN and suppressed the activity of AKT. Overall, the present study suggests that PREX2 promotes the proliferation and migration of HCC cells by inhibiting PTEN-AKT signaling.
\end{abstract}

Correspondence to: Professor Shijie Sun, Department of Hepatobiliary Surgery, Yuhuangding Hospital, 20 Yuhuangding East Road, Yantai, Shandong 264001, P.R. China

E-mail: yantaisunshijie@163.com

Key words: proliferation, phosphatidylinositol-3,4,5-trisphosphate Rac exchanger 2, hepatocellular carcinoma, migration

\section{Introduction}

Hepatocellular carcinoma (HCC) is one of the most common malignancies in humans; worldwide, the incidence of $\mathrm{HCC}$ is increasing, and HCC is the third leading cause of cancer-associated mortality (1). There has been considerable investigation concerning the molecular mechanism underlying HCC, and accumulating evidence has demonstrated that deregulating oncogenes and tumor suppressor genes are key to the development and progression of HCC (2). Therefore, the development of effective molecular targets and agents is urgently required for the treatment of HCC.

It has been well-established that the protein kinase B (AKT) signaling pathway is key in various types of human cancer, including HCC $(3,4)$. An inhibition of AKT signaling effectively suppresses HCC growth $(5,6)$. Phosphatase and tensin homolog (PTEN) encodes a major lipid phosphatase, which signals through the AKT pathway (7). PTEN acts as an inhibitor of AKT signaling, and is frequently downregulated or mutated in human cancers $(7,8)$. Therefore, upregulation of PTEN activity leads to a reduction in AKT pathway activity, and an inhibition of PTEN may result in the activation of AKT signaling (9).

Phosphatidylinositol 3,4,5-trisphosphate Rac exchanger 2 (PREX2) is a $183 \mathrm{kDa}$ protein that activates the small guanosine triphosphatase Rac, and is capable of mediating Rac signaling downstream of $\mathrm{G}$ protein-coupled receptors and AKT (10). A previous study demonstrated that PREX2 regulates Purkinje cell dendrite morphology and motor coordination (11), and it has been reported to inhibit the activity of PTEN by binding directly to PTEN via the guanine nucleotide exchange factor domain of PTEN (12). Furthermore, PREX2 may work in combination with a phosphatidylinositol-4,5-bisphosphate 3-kinase (PIP3), catalytic subunit alpha mutant to promote proliferation and transformation of cells, which is growth-factor independent (12). Knockout of PREX2 suppresses the activity of AKT and inhibits cell growth with intact PTEN (12). Previously, PREX2 has been suggested to play a role in several human cancers, including melanoma, gastric cancer and neuroblastoma (13-15). In addition, chemokine ligand 9 was identified to promote HCC cell invasion through the upregulation of PREX2, suggesting that PREX2 is involved in the regulation of HCC cell invasion (16). However, the role of 
PREX2 in the regulation of HCC cell proliferation and migration remains unclear.

The present study aimed to investigate the exact role of PREX2 in the regulation of HCC cell proliferation and migration, as well as the underlying molecular mechanism.

\section{Materials and methods}

Reagents. Dulbecco's minimum essential medium (DMEM), 3-(4,5-dimethylthiazol-2-yl)-2,5-diphenyltetrazolium bromide (MTT), fetal bovine serum (FBS), Lipofectamine ${ }^{\circledR} 2000$ and Trizol reagent were purchased from Thermo Fisher Scientific, Inc. (Waltham, MA, USA). RevertAid First Strand cDNA Synthesis kit was purchased from Fermentas (Thermo Fisher Scientific, Inc.). $\mathrm{iQ}^{\mathrm{TM}} \mathrm{SYBR}^{\circledR}$ Green Supermix was purchased from Bio-Rad Laboratories, Inc. (Hercules, CA, USA). The following primary rabbit anti-human antibodies were used: PREX2 polyclonal (catalog no., ab121462), phosphorylated (p)-PTEN monoclonal (catalog no., ab109454), PTEN monoclonal (catalog no., ab32199), p-AKT monoclonal (catalog no., ab81283), AKT monoclonal (catalog no., ab32505) and glyceraldehyde-3-phosphate dehydrogenase (GAPDH) monoclonal (catalog no., ab128915) antibodies. All antibodies, including mouse anti-rabbit secondary polyclonal immunoglobulin $\mathrm{G}$ horseradish peroxidase antibody (catalog no., ab6728), were purchased from Abcam (Cambridge, MA, USA). An enhanced chemiluminescence (ECL) kit was purchased from Pierce Biotechnology, Inc. (Rockford, IL, USA). PREX2 small interfering (si) RNA was purchased from Santa Cruz Biotechnology, Inc. (Dallas, TX, USA). PREX2 plasmid was purchased from DNASU Plasmid Repository (Tempe, AZ, USA).

Tissue specimens. The current study was approved by the Ethics Committee of Yuhuangding Hospital (Yantai, Shandong, China). A total of 15 primary HCC tissues and matched non-tumorous adjacent specimens were collected from 15 patients undergoing liver resection at the Department of Hepatobiliary Surgery at Yuhuangding Hospital. Written informed consent was obtained from each patient. The histomorphology of all the samples were confirmed by the Department of Pathology at Yuhuangding Hospital. HCC tissues were immediately snap-frozen in liquid nitrogen following surgical removal.

Cell culture. Human HCC HepG2, LH86, LMH and PLHC-1 cell lines, and normal human liver THLE-3 cell line were obtained from the American Type Culture Collection (Manassas, VA, USA). The cells were cultured in DMEM with $10 \% \mathrm{FBS}$ at $37^{\circ} \mathrm{C}$ in a humidified incubator containing $5 \% \mathrm{CO}_{2}$.

Reverse transcription-quantitative polymerase chain reaction (RT-qPCR) analysis. Total RNA was extracted from the tissue samples using Trizol reagent, according to the manufacturer's protocol. For mRNA detection, RevertAid First Strand cDNA Synthesis kit was used to convert RNA into complementary DNA, according to the manufacturer's protocol. RT-qPCR was performed using iQ SYBR Green Supermix and an Applied Biosystems 7500 thermocycler (Thermo Fisher Scientific, Inc.). The specific primer pairs were as follows: PREX2 sense,
5'-GGACTCCACGGAAACACAGTG-3' and anti-sense, 5'-GTGAGACTGCCATTCCTCTAAAA-3'; GAPDH, as a reference, sense, 5'-ACAACTTTGGTATCGTGGAAGG-3' and anti-sense, 5'-GCCATCACGCCACAGTTTC-3'. Independent experiments were repeated three times. The relative expression levels of mRNA were analyzed by use of the $2^{-\Delta \Delta \mathrm{Cq}}$ method.

Transfection of HCC cells. Lipofectamine 2000 was used to perform cell transfection, according to the manufacturer's protocol. For PREX2 functional analysis, HCC cells were transfected with PREX2 siRNA or pcDNA3.1-PREX2 plasmid.

Western blot analysis. Tissues and cell lines were solubilized in cold RIPA lysis buffer (Beyotime Institute of Biotechnology, Shanghai, China). The proteins were separated using $10 \%$ sodium dodecyl sulfate polyacrylamide gel electrophoresis and transferred onto a polyvinylidene difluoride membrane (Thermo Fisher Scientific Inc.). The polyvinylidene difluoride membrane was incubated with phosphate buffered saline containing $5 \%$ milk overnight at $4^{\circ} \mathrm{C}$. Subsequently, the polyvinylidene difluoride membrane was incubated with Tris-buffered saline and Tween 20 containing 5\% milk at room temperature for $3 \mathrm{~h}$, and incubated with rabbit anti-human PREX2 polyclonal (dilution, 1:200) and rabbit anti-human p-PTEN (dilution, 1:500), PTEN (dilution, 1:100), p-AKT, (dilution, 1:100), AKT (dilution, 1:200) and GAPDH (dilution, 1:100) monoclonal antibodies at room temperature for $3 \mathrm{~h}$. Following the incubation, the membrane was incubated again with rabbit anti-mouse secondary antibody (dilution, 1:10,000) at room temperature for $1 \mathrm{~h}$. An ECL kit was used to perform chemiluminescence detection. The relative protein expression was analyzed by Image-Pro ${ }^{\circledR}$ Plus software version 6.0 (Media Cybernetics, Inc., Rockville, MD, USA), represented as the density ratio vs. GAPDH.

Cell proliferation assay. MTT assay was used to measure cell proliferation. The cells were cultured in a 96-well plate (10,000 cells/well), each well had $100 \mu 1$ fresh serum-free medium with $0.5 \mathrm{~g} / 1 \mathrm{MTT}$. Following an incubation at $37^{\circ} \mathrm{C}$ for $0,24,48$ and $72 \mathrm{~h}$, the medium was removed by aspiration and $50 \mu 1$ dimethyl sulfoxide (Sigma-Aldrich, St. Louis, MO, USA) was added to each well. Following an incubation at $37^{\circ} \mathrm{C}$ for $10 \mathrm{~min}$, the A492 of each sample was measured using a plate reader (SM800 Microplate Reader; Shanghai Utrao Medical Instrument Co., Ltd., Shanghai, China).

Scratch assay. The cells were collected by incubation with $0.5 \%$ trypsin (Thermo Fisher Scientific Inc.) and re-suspended in DMEM containing 10\% FBS. Each well of a 24-well plate was seeded with $5 \times 10^{4}$ cells. When the cells reached $\sim 100 \%$ confluence following culturing at $37^{\circ} \mathrm{C}$ in an atmosphere of $5 \% \mathrm{CO}_{2}$, the bottom of the wells were scratched with a $10 \mu \mathrm{l}$ pipette tip. The cells were washed with serum-free medium and cultured in DMEM medium containing $10 \% \mathrm{FBS}$ at $37^{\circ} \mathrm{C}$ in an atmosphere of $5 \% \mathrm{CO}_{2}$ for $36 \mathrm{~h}$. Images of the cells were captured (DP26 Digital Camera for Microscopy; Olympus Corporation, Tokyo, Japan).

Statistical analysis. All data are presented as the mean \pm standard deviation. One-way analysis of variance were used to 

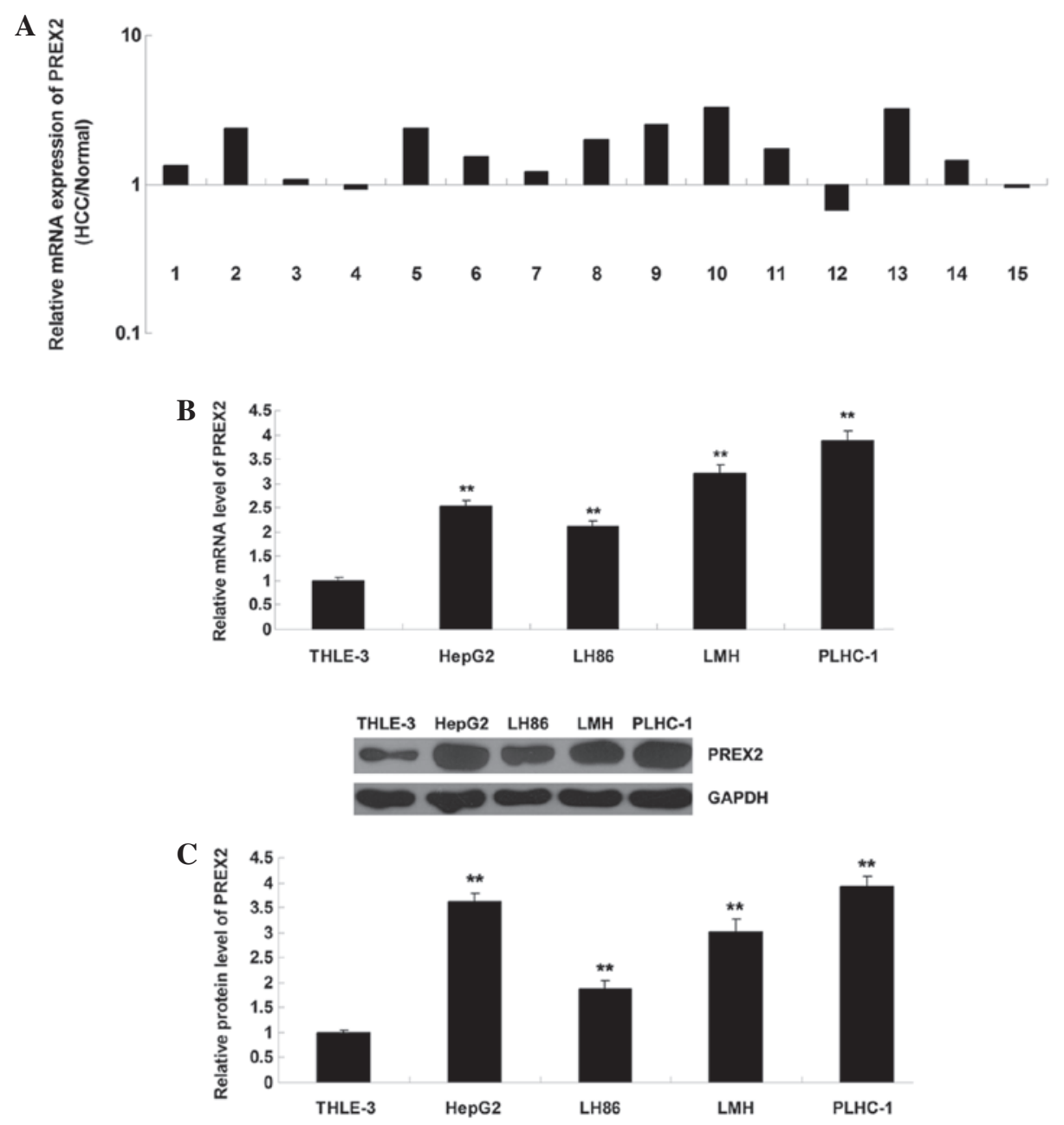

Figure 1. (A) The mRNA level of PREX2 was examined in HCC tissues and Normal tissue using reverse transcription-quantitative polymerase chain reaction. (B) The mRNA and (C) protein level of PREX2 was determined using western blot analysis in various HCC cell lines. The human normal liver THLE-3 cell line was used as a control. "** $\mathrm{P}<0.01$ vs. control. mRNA, messenger RNA; PREX2, phosphatidylinositol-3,4,5-trisphosphate Rac exchanger 2; HCC, hepatocellular carcinoma; Normal, matched adjacent normal tissue.

analyze the data using SPSS software version 17 (SPSS, Inc., Chicago, IL, USA). $\mathrm{P}<0.05$ was considered to indicate a statistically significant difference.

\section{Results}

PREX2 was upregulated in HCC tissues. The present study examined the expression of PREX2 in HCC tissues and human HCC HepG2, LH86, LMH and PLHC-1 cell lines. As revealed in Fig. 1A, the expression of PREX2 was increased in $\mathrm{HCC}$ tissues compared with the normal matched adjacent tissues. Fig. 1B and C demonstrate that the mRNA and protein expression level of PREX2 was also significantly increased in the HCC cell lines compared with the normal human liver THLE-3 cells. These findings suggest that deregulation of PREX2 is implicated in HCC. Since PLHC-1 cells demonstrated the most significant upregulation of PREX2, the present study used this cell line in subsequent experiments.

Upregulation of PREX2 promoted the proliferation of HCC cells. The present study investigated the role of PREX2 in the regulation of HCC proliferation in vitro in more detail. The current study transfected PLHC-1 cells with PREX2 plasmid or PREX2 siRNA to modulate the expression level of PREX2. Following transfection, RT-qPCR and western blot analysis were used to determine the mRNA and protein levels of PREX2 in PLHC-1 cells. The results demonstrated that the mRNA and protein levels of PREX2 were upregulated in PLHC-1 cells transfected with PREX2 plasmid, and reduced in PLHC-1 cells transfected with PREX2 siRNA compared with the control cells (control cells, non-transfected PLHC-1 cells; Fig. 2A and B).

Subsequently, anMTT assay was performed to determine cell proliferation in PLHC-1 cells transfected with PREX2 plasmid or PREX2 siRNA. As revealed in Fig. 2C, the proliferation of PLHC-1 cells was significantly reduced following knockdown of PREX2, and the proliferation was notably increased following overexpression of PREX2 compared with the control cells. Therefore, this suggests that PREX2 serves an oncogenic role in the regulation of HCC cell proliferation.

Upregulation of PREX2 enhanced HCC cell migration. A scratch assay was performed to determine cell migration in PLHC-1 cells transfected with PREX2 plasmid or PREX2 siRNA (Fig. 3A). Following the inhibition of PREX2 expression, the migration of PLHC-1 cells was 

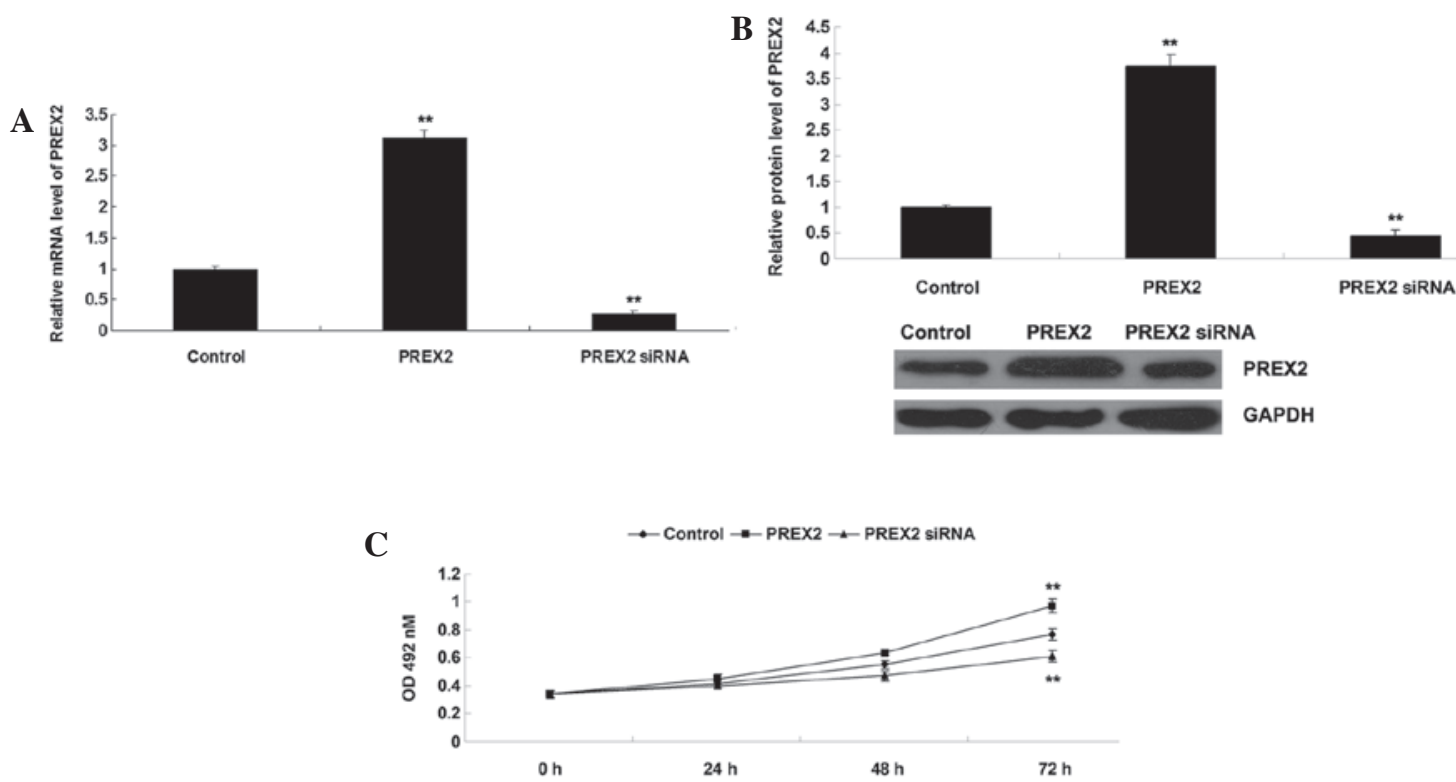

Figure 2. (A) The mRNA and (B) protein levels of PREX2 were determined using reverse transcription-quantitative polymerase chain reaction and western blot analysis in human hepatocellular carcinoma PLHC-1 cells transfected with PREX2 plasmid or PREX2 small interfering RNA. (C) 3-(4,5-dimethylthiazol-2-yl)-2,5-diphenyltetrazolium bromide assay was used to determine cell proliferation. ${ }^{* *} \mathrm{P}<0.01 \mathrm{vs}$. control. mRNA, messenger RNA; PREX2, phosphatidylinositol-3,4,5-trisphosphate Rac exchanger 2; Control, non-transfected human hepatocellular carcinoma PLHC-1 cells; OD, optical density.

A
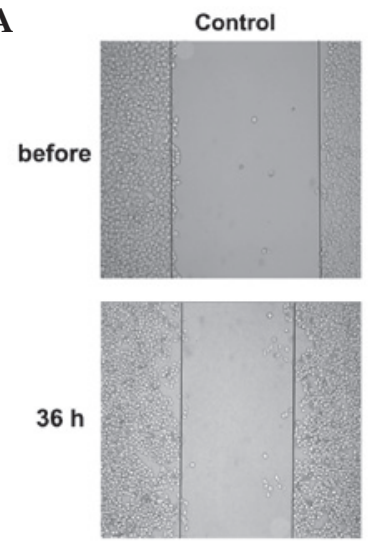

B
PREX2
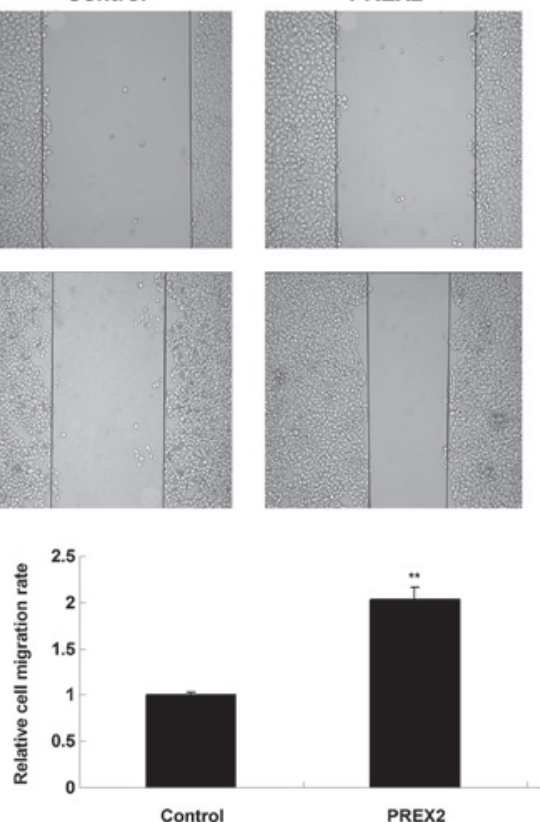

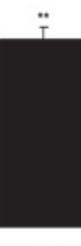

PREX2
PREX2 SiRNA
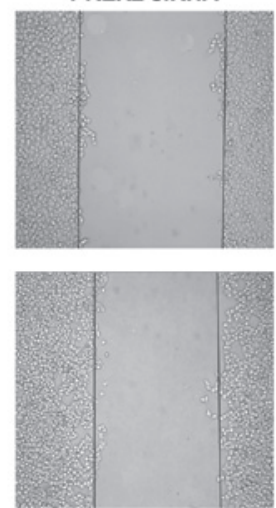

Figure 3. Scratch assay was performed to determine cell migration in human hepatocellular carcinoma PLHC-1 cells transfected with PREX2 plasmid or PREX2 small interfering RNA. (A) Image of Scratch assay, and (B) quantification of scratch assay. ${ }^{* *} \mathrm{P}<0.01$ vs. control. PREX2, phosphatidylinositol-3,4,5-trisphosphate Rac exchanger 2; Control, non-transfected human hepatocellular carcinoma PLHC-1 cells.

significantly reduced compared with the control cells (Fig. 3B). Following overexpression of PREX2, the migratory capacity of PLHC-1 cells was notably upregulated compared with the control cells (Fig. 3B). Accordingly, this suggests that PREX2 may serve an oncogenic role in the regulation of $\mathrm{HCC}$ cell migration.

PREX2 inhibited PTEN activity and activated AKT signaling in HCC cells. PREX2 inhibits the activity of PTEN, and activates AKT signaling (16). Accordingly, the present study investigated the activity of PTEN and AKT signaling pathways in PLHC-1 cells transfected with PREX2 plasmid or PREX2 siRNA by western blot analysis (Fig. 4A). The findings demonstrated that p-PTEN expression levels were increased in PLHC-1 cells transfected with PREX2 plasmid, indicating that the activity of PTEN was reduced. By contrast, the activity of PTEN was upregulated in PLHC-1 cells following knockdown of PREX2. Furthermore, the level of p-AKT was 
A

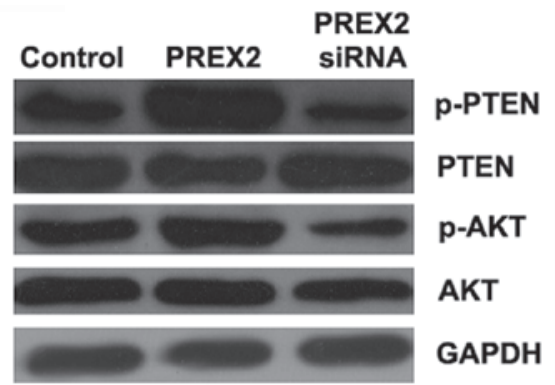

B

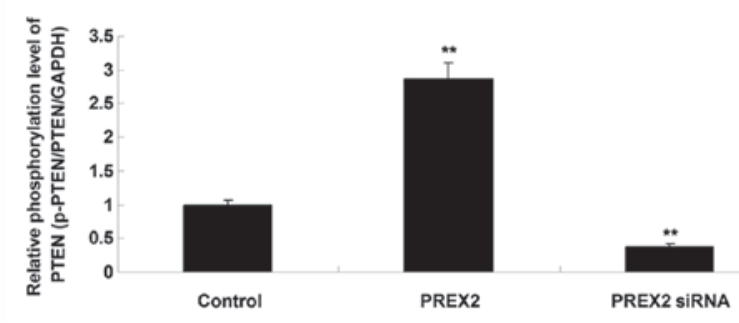

\section{C}

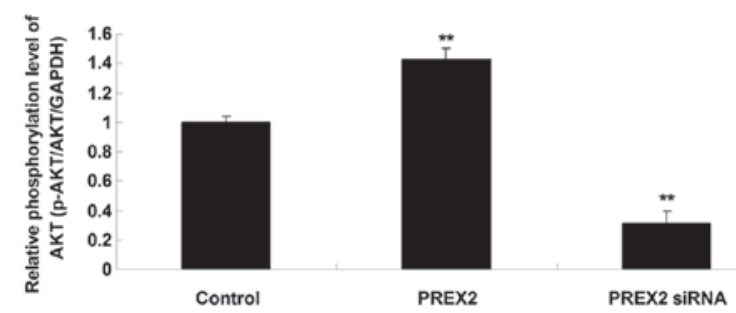

Figure 4. (A) Western blot analysis was performed to determine the protein expression of p-PTEN, PTEN, p-AKT, and AKT in human hepatocellular carcinoma PLHC-1 cells transfected with PREX2 plasmid or PREX2 siRNA. GAPDH was used as a reference. Relative phosphorylation level of (B) PTEN and (C) AKT. ${ }^{* *} \mathrm{P}<0.01$ vs. control. p, phosphorylated; PTEN, phosphatase and tensin homolog deleted on chromosome 10 AKT, protein kinase B; PREX2, phosphatidylinositol-3,4,5-trisphosphate Rac exchanger 2; siRNA, small interfering RNA; Control, non-transfected human hepatocellular carcinoma PLHC-1 cells; GAPDH, glyceraldehyde 3-phosphate dehydrogenase.

increased in PLHC-1 cells with an overexpression of PREX2, indicating that the activity of the AKT signaling pathway was upregulated (Fig. 4C; $\mathrm{P}<0.01$ ). By contrast, knockdown of PREX2 downregulated the activity of the AKT signaling pathway in PLHC-1 cells (Fig. 4C; $\mathrm{P}<0.01$ ). Therefore, these findings indicate that PREX2 inhibits PTEN activity and activates AKT signaling in HCC cells.

\section{Discussion}

The identification of effective molecular targets is urgently required for the treatment of HCC (17). The present study demonstrated that the expression of PREX2 was increased in $\mathrm{HCC}$ tissues compared with matched normal adjacent tissues. In addition, PREX2 was significantly upregulated in HCC cell lines compared with a normal liver cell line. The present in vitro study revealed that PREX2 promoted the proliferation and migration of HCC cells by modulating PTEN-AKT signaling.

PREX2 is a guanine nucleotide exchange factor that acts on the guanosine triphosphatase Rac, and PREX2 has been identified as an inhibitor of PTEN (18). PTEN negatively regulates intracellular levels of (PIP3) in cells and functions as a tumor suppressor gene by negatively regulating the AKT signaling pathway $(19,20)$. Therefore, PREX2-induced inactivation of PTEN may lead to an accumulation of PIP3, which may increase the activity of the downstream AKT signaling pathway (12).

As the AKT signaling pathway acts as a key regulator in tumorigenesis, PREX2 may be crucial in human cancers $(2,21)$. In an independent extension cohort of 107 human melanomas, whole-genome sequencing identified PREX2 as a significantly mutated gene with a mutation frequency of $\sim 14 \%$ (15). These mutations consisted of non-synonymous PREX2 mutations and truncations, which were distributed along the entire length of the PREX2 gene. Furthermore, ectopic expression of mutant PREX2, which was present in patients with melanomas, accelerated the tumor formation of immortalized human melanocytes in vivo (15). In addition, overexpression of PREX2 has been revealed to be associated with the poor overall survival of patients with breast cancer (22). The present study identified that the expression of PREX2 was upregulated in HCC tissues and cell lines compared with adjacent normal tissues and normal liver cell lines. Lan et al (16) demonstrated that high mRNA levels of PREX2 were associated with poor differentiation, portal vein invasion and metastasis of cells and qualitative hepatitis B surface antigen in 45 HCC tissue specimens. In addition, the authors demonstrated that the inhibition of PREX2 by siRNA reduced the invasion ability of liver cancer cells (16). Therefore, PREX2 may be critical in HCC development.

PREX2 is involved in several types of human cancer, including HCC; however, the exact role of PREX2 in the regulation of $\mathrm{HCC}$ cell proliferation and migration has not been elucidated. In the present study, HCC cells were transfected with PREX2-specific siRNA or PREX2 plasmid to downregulate or upregulate PREX2 expression, respectively. It was observed that knockdown of PREX2 significantly suppressed the proliferation and migration of HCC cells, and overexpression of PREX2 notably enhanced HCC cell proliferation and migration. Consequently, the evidence in the present study suggests that PREX2 may play a promoting role in the regulation of HCC growth and metastasis. It has been reported that PREX2 stimulates cell proliferation by inhibiting PTEN and stimulating downstream AKT signaling (23). Chen et al (14) revealed that knockdown of PREX2 inhibited the proliferation, migration and invasion of neuroblastoma cells by activating PTEN and suppressing the AKT signaling pathway. Similarly, Guo et al (13) reported that an inhibition of PREX2 suppressed the proliferation and clonogenicity of 
gastric cancer cells, and induced cell apoptosis and a cell cycle arrest at G1-S phase, by activating PTEN and inhibiting AKT activity. The current study identified that inhibiting the PREX2 expression increased the activity of PTEN and reduced the activity of AKT, and an overexpression of PREX2 upregulated the PTEN activity and inactivated AKT, suggesting that PREX2 promotes the proliferation and migration in HCC cells by modulating PTEN-AKT signaling.

In summary, the present study demonstrated that PREX2 was upregulated in HCC tissues and cell lines and exerted promoting effects on the proliferation and migration of $\mathrm{HCC}$ cells by inhibiting PTEN activity, and therefore activating AKT signaling. Consequently, PREX2 may be a potential target for the treatment of HCC.

\section{References}

1. Zhu AX: Molecularly targeted therapy for advanced hepatocellular carcinoma in 2012: Current status and future perspectives. Semin Oncol 39: 493-502, 2012.

2. Psyrri A, Arkadopoulos N, Vassilakopoulou M, Smyrniotis V and Dimitriadis G: Pathways and targets in hepatocellular carcinoma. Expert Rev Anticancer Ther 12: 1347-1357, 2012.

3. Dimitrova V and Arcaro A: Targeting the PI3K/AKT/mTOR signaling pathway in medulloblastoma. Curr Mol Med 15: 82-93, 2015.

4. Ohta K, Hoshino H, Wang J, Ono S, Iida Y, Hata K, Huang SK, Colquhoun S and Hoon DS: MicroRNA-93 activates c-Met/PI3K/Akt pathway activity in hepatocellular carcinoma by directly inhibiting PTEN and CDKN1A. Oncotarget 6: 3211-3224, 2015.

5. Wang Y, Hu C, Cheng J, Chen B, Ke Q, Lv Z, Wu J and Zhou Y: MicroRNA-145 suppresses hepatocellular carcinoma by targeting IRS1 and its downstream Akt signaling. Biochem Biophys Res Commun 446: 1255-1260, 2014.

6. Wang HY, Yang SL, Liang HF and Li CH: HBx protein promotes oval cell proliferation by up-regulation of cyclin D1 via activation of the MEK/ERK and PI3K/Akt pathways. Int J Mol Sci 15: 3507-3518, 2014

7. Carnero A and Paramio JM: The PTEN/PI3K/AKT pathway in vivo, cancer mouse models. Front Oncol 4: 252, 2014.

8. Eng C: PTEN: One gene, many syndromes. Hum Mutat 22: 183-198, 2003
9. Hers I, Vincent EE and Tavaré JM: Akt signalling in health and disease. Cell Signal 23: 1515-1527, 2011.

10. Donald S, Hill K, Lecureuil C, Barnouin R, Krugmann S, John Coadwell W, Andrews SR, Walker SA, Hawkins PT, Stephens LR and Welch HC: P-Rex2, a new guanine-nucleotide exchange factor for Rac. FEBS Lett 572: 172-176, 2004.

11. Donald S, Humby T, Fyfe I, Segonds-Pichon A, Walker SA, Andrews SR, Coadwell WJ, Emson P, Wilkinson LS and Welch HC: P-Rex 2 regulates Purkinje cell dendrite morphology and motor coordination. Proc Natl Acad Sci USA 105: 4483-4488, 2008.

12. Fine B, Hodakoski C, Koujak S, Su T, Saal LH, Maurer M, Hopkins B, Keniry M, Sulis ML, Mense S, et al: Activation of the PI3K pathway in cancer through inhibition of PTEN by exchange factor P-REX2a. Science 325: 1261-1265, 2009.

13. Guo B, Liu L, Yao J, Ma R, Chang D, Li Z, Song T and Huang C: miR-338-3p suppresses gastric cancer progression through a PTEN-AKT axis by targeting P-REX2a. Mol Cancer Res 12: 313-321, 2014.

14. Chen X, Pan M, Han L, Lu H, Hao X and Dong Q: miR-338-3p suppresses neuroblastoma proliferation, invasion and migration through targeting PREX2a. FEBS Lett 587: 3729-3737, 2013.

15. Berger MF, Hodis E, Heffernan TP, Deribe YL, Lawrence MS, Protopopov A, Ivanova E, Watson IR, Nickerson E, Ghosh P, et al: Melanoma genome sequencing reveals frequent PREX2 mutations. Nature 485: 502-506, 2012.

16. Lan X, Xiao F, Ding Q, Liu J, Liu J, Li J, Zhang J and Tian DA: The effect of CXCL9 on the invasion ability of hepatocellular carcinoma through up-regulation of PREX2. J Mol Histol 45: 689-696, 2014.

17. Hung $\mathrm{CH}$, Chiu YC, Chen $\mathrm{CH}$ and $\mathrm{Hu} \mathrm{TH}$ : MicroRNAs in hepatocellular carcinoma: Carcinogenesis, progression, and therapeutic target. Biomed Res Int 2014: 486407, 2014.

18. Hodakoski C, Hopkins BD, Barrows D, Mense SM, Keniry M, Anderson KE, Kern PA, Hawkins PT, Stephens LR and Parsons R: Regulation of PTEN inhibition by the pleckstrin homology domain of P-REX2 during insulin signaling and glucose homeostasis. Proc Natl Acad Sci USA 111: 155-160, 2014.

19. Salmena L, Carracedo A and Pandolfi PP: Tenets of PTEN tumor suppression. Cell 133: 403-414, 2008.

20. Trotman LC, Niki M, Dotan ZA, Koutcher JA, Di Cristofano A, Xiao A, Khoo AS, Roy-Burman P, Greenberg NM, Van Dyke T, et al: Pten dose dictates cancer progression in the prostate. PLoS Biol 1: E59, 2003.

21. Oishi N, Yamashita T and Kaneko S: Molecular biology of liver cancer stem cells. Liver Cancer 3: 71-84, 2014.

22. Pandiella A and Montero JC: Molecular pathways: P-Rex in cancer. Clin Cancer Res 19: 4564-4569, 2013.

23. Leslie NR: P-REX2a driving tumorigenesis by PTEN inhibition. Sci Signal 2: pe68, 2009. 